\title{
OBSERVATIONAL STUDY OF DIAGNOSTIC ACCURACY OF MODIFIED ALVARADO SCORE AND ULTRASONOGRAPHY IN ACUTE APPENDICITIS IN ADULTS
}

\author{
Varun Dogra', Shakeel Ahmad Mir², Syed Mushtaq Ahmad Shah', Ishfaq Ahmed Gilkar", Hanief Mohamed Dar ${ }^{5}$
}

${ }^{1}$ Senior Resident, Department of Surgery, GMC Jammu, Bakshi Nagar, Jammu and Kashmir, India.

${ }^{2}$ Associate Professor, Department of Surgery, GMC, Srinagar, Karan Nagar, Jammu and Kashmir, India.

3 Professor, Department of Surgery, GMC, Srinagar, Karan Nagar, Jammu and Kashmir, India.

${ }^{4}$ Senior Resident, Department of Surgery, GMC, Srinagar, Karan Nagar, Jammu and Kashmir, India.

${ }_{5}^{5}$ Senior Resident, Department of Surgery, GMC, Srinagar, Karan Nagar, Jammu and Kashmir, India.

\section{ABSTRACT}

\section{BACKGROUND}

Vermiform appendix is a narrow, worm-shaped tube which springs from the posteromedial wall of the caecum. It participates in the secretion of immunoglobulin, particularly IgA. Acute appendicitis is the most common surgical emergency with an annual incidence in the USA of 9.38 per 100,000. Arriving at the correct diagnosis is essential; however, a delay may allow progression to perforation and significantly increased morbidity and mortality.

Aims and Objectives- We aim to study the efficacy of Modified Alvarado Scoring system versus Ultrasonography in adults to decrease the number of negative appendectomies.

\section{MATERIALS AND METHODS}

The study "Observational Study of Diagnostic Accuracy of Modified Alvarado Score and Ultrasonography in Acute Appendicitis in Adults" is a prospective observational study, which comprised of 200 patients above the age of 16, admitted in the Department of Surgery, Government Medical College, Srinagar with a provisional diagnosis of Acute Appendicitis. Sensitivity, Specificity, Predictive value for Positive and Negative tests and Accuracy of each diagnostic modality were worked out and compared with histopathological outcome.

\section{RESULTS}

We found that Modified Alvarado Score had the best Sensitivity, Specificity, Positive Predictive Value and Negative Predictive Value. However, Specificity of Total Leukocyte Count was equal to that of Modified Alvarado, but it had the least sensitivity and accuracy. Ultrasonography has the highest accuracy, which was comparable to that of Modified Alvarado Score. Ultrasonography on the other hand had the least Specificity, Positive Predictive Value and Negative Predictive Value.

\section{CONCLUSION}

Modified Alvarado and ultrasonography should be used together in surgical emergency for diagnosing cases of acute appendicitis.

\section{KEYWORDS}

Acute Appendicitis, Modified Alvarado Score, Appendix, Ultrasonography, Leucocytosis.

HOW TO CITE THIS ARTICLE: Dogra V, Mir SA, Shah SMA, et al. Observational study of diagnostic accuracy of modified Alvarado score and ultrasonography in acute appendicitis in adults. J. Evolution Med. Dent. Sci. 2018;7(05):667-671, D0I: 10.14260/jemds/2018/151

\section{BACKGROUND}

The Vermiform appendix, present only in human beings, certain arthropod, apes and the wombat (a nocturnal, burrowing Australian marsupial), dates to its discovery as early as the Egyptian civilisation (3000 BC). It was Verneys who in 1710 coined the term "Vermiform appendix," vermiform meaning worm-like.[1] Vermiform appendix is a narrow, worm-shaped tube which springs from the posteromedial wall of the caecum. It participates in the secretion of immunoglobulin, particularly IgA. Although, an important component of gut associated lymphoid tissue (GALT) system, its function is not essential and

'Financial or Other Competing Interest': None.

Submission 20-12-2017, Peer Review 11-01-2018,

Acceptance 19-01-2018, Published 29-01-2018.

Corresponding Author:

Dr. Varun Dogra,

Village - Daskal, Post office-Akhnoor,

District-Jammu-181201,

Jammu and Kashmir.

E-mail: drdogravarun@gmail.com

DOI: $10.14260 /$ jemds $/ 2018 / 151$

\section{(c) (i) $९$}

appendectomy is not associated with any predisposition to sepsis or any other manifestation of immune compromise.[2,3,4] Acute appendicitis (Photo 1) occurs when the appendiceal lumen is obstructed, leading to fluid accumulation, luminal distention, inflammation and finally perforation. $[5,6,7,8]$ Acute Appendicitis is the most common surgical emergency with an annual incidence in the USA of


management may differ. The mainstay of treatment for majority of patients remains surgery either by an open or by laparoscopic approach. Some of the signs and symptoms can be subtle to both the clinician and the patient, and may not be present in all instances. Arriving at the correct diagnosis is essential; however, a delay may allow progression to perforation and significantly increased morbidity and mortality. Incorrectly diagnosing a patient with Appendicitis, although not catastrophic, often subjects the patient to an unnecessary operation. A clinical decision to operate leads to the removal of a normal appendix in $15 \%$ to $30 \%$ of cases (although the figure may be higher or lower in certain demographic groups).[10] Methods advocated to assist in the diagnosis of Appendicitis include Scoring Systems, 
Ultrasonography, Computed Tomography, Magnetic Resonance Imaging and Laparoscopy. Several scoring systems have been used globally for early diagnosis of acute appendicitis. One of the most practical scoring systems is the Modified Alvarado Scoring System (MASS), developed by Kalan. It is a 9-point scoring system (Table 1) based on history, physical examination and some laboratory investigations that helps in increasing the accuracy of preoperative diagnosis and thus reducing negative appendectomy rate. Patients with a Modified Alvarado Score of 7 or more were recommended for surgery.[11,12] In the past, ultrasonography has been gradually introduced as a diagnostic imaging procedure for Acute Appendicitis.[13] It has been claimed that Ultrasonography dramatically reduces the number of appendectomy in patients without appendicitis.[14] Its via histopathological evidence that we can confirm a diagnosis of Acute Appendicitis after surgery.

\section{Aims and Objectives}

We aim to study the efficacy of Modified Alvarado Scoring System versus Ultrasonography in adults to decrease the number of negative appendectomies.

\section{MATERIALS AND METHODS}

The study "Observational Study of Diagnostic Accuracy of Modified Alvarado Score and Ultrasonography in Acute Appendicitis in Adults" is a prospective observational study, which comprised of 200 patients admitted in the Department of Surgery, Government Medical College, Srinagar with a provisional diagnosis of Acute Appendicitis. Only those patients in which surgery was planned on clinical grounds were included in the study. All patients selected were above 16 years of age and either sex was evaluated on the basis of predetermined proforma, which included a detailed history from patients or attendants, clinical examination, laboratory investigations and high-resolution sonography.

\section{Following Patients were excluded from the Study-}

- Patients unfit for surgical intervention.

- Patients below the age of 16 years.

- Patients having uncorrected coagulopathy.

- Patients admitted for interval appendicectomy following recurrent appendicitis, appendicular abscess, appendicular mass previously treated conservatively.

A provisional diagnosis of Acute Appendicitis was made in patients having classical history of pain starting in the epigastrium or umbilical area and getting shifted and localised to the right iliac fossa with signs of tenderness at McBurney's point with or without localised guarding, rigidity, percussion or rebound tenderness. For the diagnosis of Acute Appendicitis, complete blood counts and high-resolution ultrasonography were done pre-operatively. Urine analysis was done routinely to rule out any urinary tract infections. Other relevant and necessary investigations for undergoing surgery under General or Spinal Anaesthesia were also done.

Total leucocyte count and differential leucocyte count was estimated using automated analysers in our institution. The upper limits of total leucocyte count and neutrophil percentage were taken as $10.5 \mathrm{X} 109 / \mathrm{L}$ and $75 \%$ respectively. Based on history, clinical examination and laboratory investigations, Modified Alvarado Score was calculated as shown.

Ultrasonography was performed on all these patients in radiodiagnosis department. Sonography was done by $7.5 \mathrm{MHz}$ probe for radiological signs of acute appendicitis as well as to rule out other possible causes of right lower abdominal pain, especially in females (Photo 2). Diagnosis was based on following major criteria: Visualisation of non-compressible, aperistaltic, painful appendix with an outer diameter of more than $6 \mathrm{~mm}$ presenting with a "target like" cross-sectional view and a tubular appearance with a blind ending tip on the longitudinal scan, surrounded by echogenic inflamed fat (Photo 3). Additional criteria were appendicolith and the absence of gas in the lumen. Criteria of less importance were free peritoneal fluid and enlarged regional lymph nodes.[15,16,17] The criteria for perforation were disruption of the wall and/or presence of an abscess. If the appendix was not visualised in addition to having no other findings, the ultrasonographic examination was considered negative. Appendectomy was performed in all cases of clinically diagnosed acute appendicitis. In all these patients, informed and written consent was taken in local language prior to surgery. All the patients were given prophylaxis for Tetanus. Patients were also given antibiotics before surgery. Removed appendices were subjected to histopathological examination, which was taken as gold standard and according to which the diagnosis was confirmed or excluded. Sensitivity, Specificity, Predictive value for Positive and Negative tests and Accuracy of each diagnostic modality were worked out and compared with histopathological outcome.

\section{RESULTS}

This study was conducted in Department of Surgery, Government Medical College, Srinagar from June 2014 to June 2016 on 200 patients with diagnosis of Acute Appendicitis. Patients of either sex 16 years and above were included in the study. The observation and results are as follows.

\section{Age and Sex Distribution}

Out of the total 200 subjects, males comprised of 122 (61\%) subjects, while females comprised of 78 (39\%) subjects (Figure 1). Highest number of subjects were seen in age group of 16 - 20 years in both males (38 subjects) and females (34 subjects), while lowest numbers were seen in age group of 41 - 50 years in both $(7$ subjects in males and 3 subjects in females) sexes. Males comprised of 61 percent of subjects, while females comprised of 39 percent of subjects

\section{Clinical Features}

The most common symptom in acute appendicitis was pain followed by nausea and vomiting, but the most specific symptom was migratory pain. Most common sign was tenderness in right lower abdomen, which was more or less present in every subject (Figure 2).

\section{Total Leukocyte Count}

We in our study found that leucocyte count had a sensitivity of $75 \%$, specificity of $93.55 \%$, positive predictive value of $98.46 \%$, negative predictive value of $57 \%$ and accuracy of $78.5 \%$. 


\section{Clinical Assessment}

Patients were categorised into three categories based on Modified Alvarado Scoring: Those with score of $1-4$ in Category One. Those with score of 5 - 7 in Category Two and those with core of greater than 7 in Category Three.

\section{Comparison of Modified Alvarado with Histopathological Analysis}

Category 1 were considered Modified Alvarado Negative, while Category 2 and 3 were considered Modified Alvarado Positive. It was found that Modified Alvarado Scoring had Sensitivity of $88.76 \%$, Specificity of $93.55 \%$, Positive Predictive Value of $98.68 \%$, Negative Predictive Value of $60.42 \%$ and Accuracy of $84.5 \%$.

\section{High Resolution Ultrasonography}

It was seen in our study that USG had Sensitivity of $85.20 \%$, Specificity of $83.87 \%$, Positive Predictive Value of $96.64 \%$, Negative Predictive Value of $50.98 \%$ and Accuracy of $85.00 \%$.

\section{Relative Accuracy of Various Diagnostic Modalities}

In the end, we compared the relative Sensitivity, Specificity, Positive Predictive Value, Negative Predictive Value and Accuracy of the various diagnostic modalities (Table 2). From the table, we find that Modified Alvarado Score had the best Sensitivity, Specificity, Positive Predictive Value and Negative Predictive Value. However, Specificity of Total Leukocyte Count was equal to that of Modified Alvarado, but it had the least sensitivity and accuracy. Ultrasonography has the highest accuracy which was comparable to that of Modified Alvarado Score. Ultrasonography on the other hand had the least Specificity Positive Predictive Value and Negative Predictive Value.

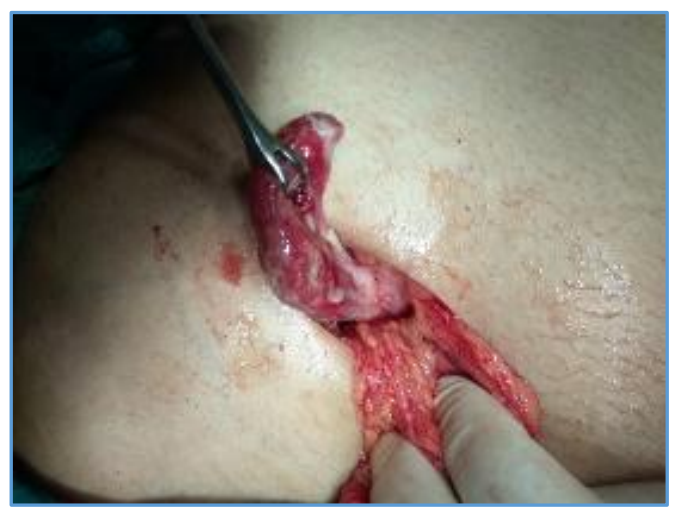

Photo 1. Acute Appendicitis

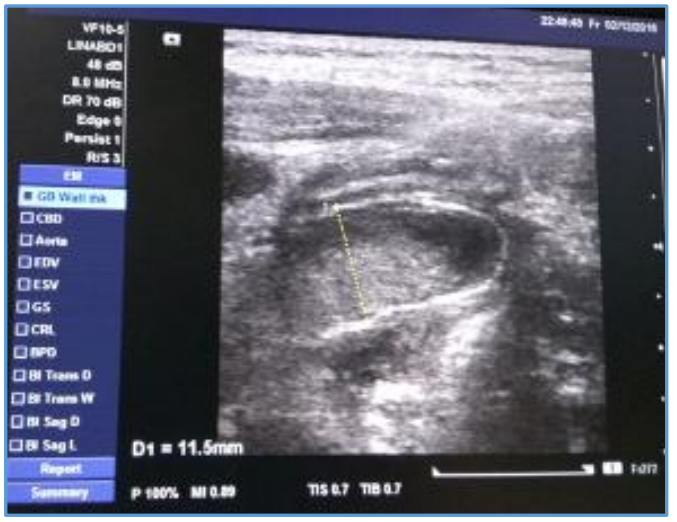

Photo 2. Increased Diameter in Acute Appendicitis

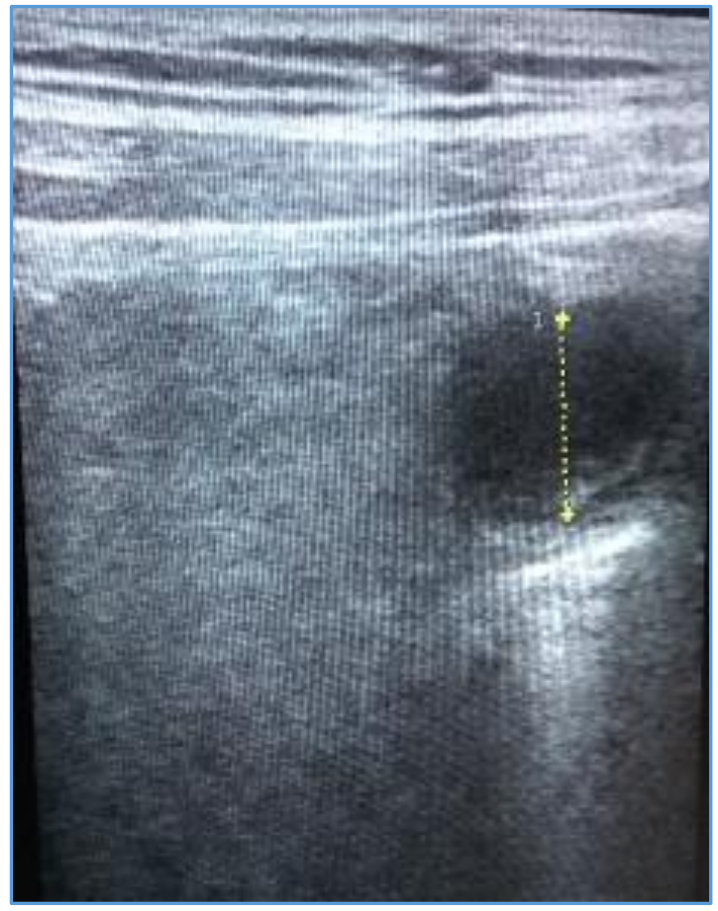

Photo 3. Target Sign in Acute Appendicitis

\begin{tabular}{|c|c|}
\hline \multicolumn{1}{|c|}{ Manifestations } & Value \\
\hline Symptoms & 1 \\
\hline a). Migration of pain & 1 \\
\hline b). Anorexia & 1 \\
\hline c). Nausea and/or vomiting & 2 \\
\hline Signs & 1 \\
\hline a). Right lower quadrant tenderness & 1 \\
\hline b). Rebound & 2 \\
\hline c). Elevated temperature laboratory value & 9 \\
\hline a). Leucocytosis & \\
\hline \multicolumn{2}{|c|}{ Total Points } \\
\hline \multicolumn{2}{|c|}{ Table 1. Criteria for Modified Alvarado Score } \\
\hline
\end{tabular}

\begin{tabular}{|c|c|c|c|}
\hline Parameters & TLC & MAS & USG \\
\hline Sensitivity & $75 \%$ & $88.76 \%$ & $85.20 \%$, \\
\hline Specificity & $93.55 \%$ & $93.55 \%$ & $83.87 \%$ \\
\hline PPV & $98.46 \%$ & $98.68 \%$ & $96.64 \%$, \\
\hline NPV & $57.0 \%$ & $60.42 \%$ & $50.98 \%$ \\
\hline Accuracy & $78.5 \%$ & $84.5 \%$ & $85.00 \%$ \\
\hline \multicolumn{4}{|c|}{ Table 2. Relative Diagnostic Accuracy } \\
of Various Modalities \\
\hline
\end{tabular}

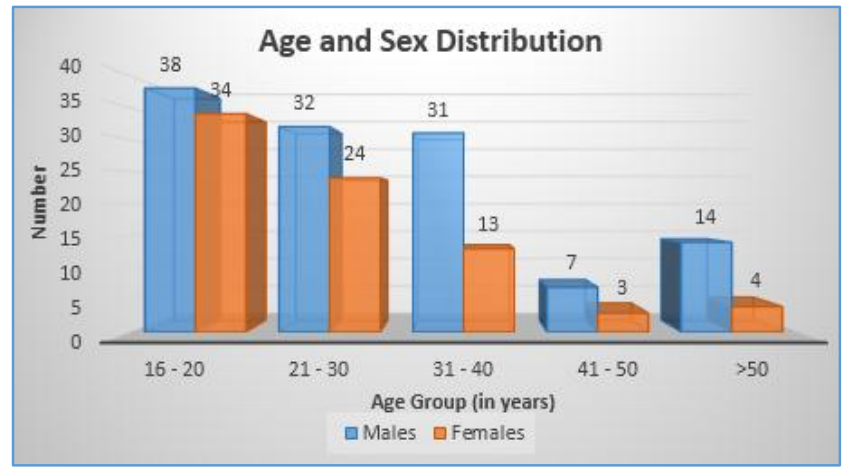

Figure 1. Age and Sex Distribution 


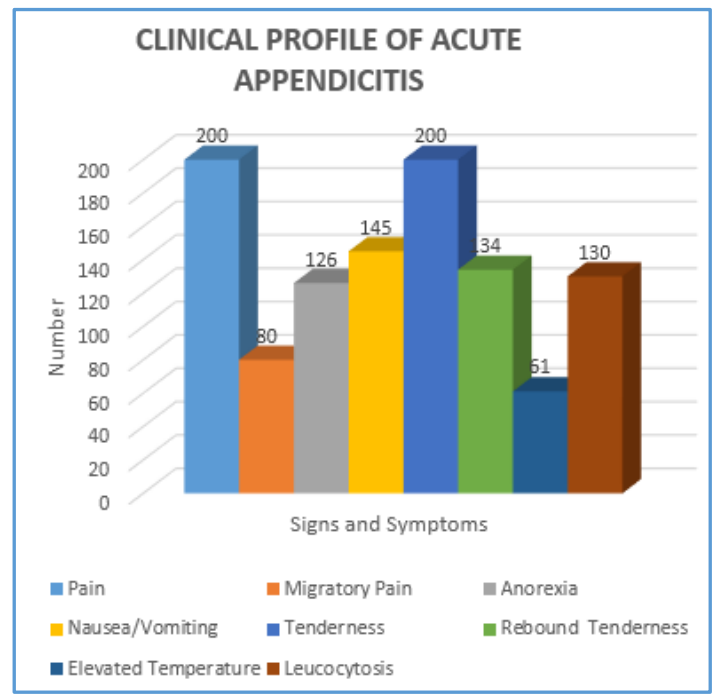

Figure 2. Clinical Profile of Acute Appendicitis

\section{DISCUSSION}

Acute Appendicitis is one of the most common surgical illness that we face in surgical emergencies. While underdiagnoses will lead to more chances of complications, an over diagnosis leads to negative appendectomies. As such it becomes very important for a surgeon to accurately diagnose and treat cases of acute appendicitis. It is also essential to use investigations judiciously so as to minimise the burden on the hospital resources as well as on the patients, yet it should not compromise on the correct diagnosis and adequate treatment. Keeping all these things in mind we performed a study in the Postgraduate Department of General Surgery, Government Medical College, Srinagar, from May 2014 to October 2016. Following things were observed during the study

- Acute appendicitis was seen predominantly in males with highest number seen in younger age group. Sex distribution of the patients approached a male-to-female ratio of 3: 2. Also it was observed that incidence of acute appendicitis decreased with increasing age.

- The most common complaint that patients of acute appendicitis presented with was pain, which was present in every patient. However, migration of pain proved to be the most specific symptom. Most common sign seen in patients of acute appendicitis was tenderness followed by rebound tenderness at the McBurney's Point.

- Among laboratory investigations the most important investigation was the total leukocyte count, which had a good specificity but an average sensitivity. However, when used along with signs and symptoms proved to be an excellent marker of acute appendicitis.

- Modified Alvarado Scoring was performed in all patients, which showed a high sensitivity and specificity in diagnosing cases of Acute Appendicitis. It also showed an excellent Positive Predictive Value; however, negative predictive value was low. Modified Alvarado Score had a good accuracy approaching $85 \%$.

All the patients were also subjected to Ultrasonography prior to surgical intervention and it was found that Ultrasonography had a good sensitivity, specificity, positive predictive value and accuracy in diagnosing a case of acute appendicitis. However, it lacked specificity and it had a low negative predictive value.

\section{CONCLUSION}

This study titled "Observational Study of Diagnostic Accuracy of Modified Alvarado Score and Ultrasonography in Acute Appendicitis in Adults" was done in Government Medical College, Srinagar for a period extending from May 2014 to October 2016 comprised of 200 subjects, subjected to emergency appendectomy after a detailed history, examination and ultrasonography. The study showed that Modified Alvarado Score had excellent Diagnostic Accuracy with high sensitivity and specificity. Ultrasonography had a good sensitivity and an average specificity which was less than that of Modified Alvarado Score. However, when both were used together in diagnosing cases of acute appendicitis, they reduced the rate of negative appendectomies to a large extent. Thus, it is summarised that both Modified Alvarado and ultrasonography should be used together in surgical emergency for diagnosing cases of acute appendicitis.

\section{REFERENCES}

[1] Herrinton JL. The Vermiform appendix: its surgical history. Contemp Surg 1991;39:36-44.

[2] Fitz RH. Persistent omphalo-mesenteric remains: their importance in the causation of intestinal duplication, cyst formation and obstruction. Am J Med Sci 1884;88:30-57.

[3] Buschard K, Kjaeldgaard A. Investigation and analysis of the position fixation, length and embryology of the vermiform appendix. Acta Chir Scand 1973;139(3):293-8.

[4] Skandalakis JE, Gray SW, Ricketts R. The colon and rectum. In: Skandalakis JE, Gray SW. eds. Embryology for surgeons. Baltimore: Williams and Wilkins 1994: p. 242.

[5] Birnbaum BA, Wilson SR. Appendicitis at the millennium. Radiology 2000;215(2):337-48.

[6] Berry J, Malt RA. Appendicitis near its centenary. Ann Surg 1984;200(5):567-75.

[7] Heaston DR, McClellan JS, Heaston DK. Community hospital experience in $600+$ consecutive patients who underwent unenhanced helical CT for suspected appendicitis. AJR 2000;174:53.

[8] Rao PM, Rhea JT, Novelline RA. Helical CT of appendicitis and diverticulitis. Radiol Clin North Am 1999;37(5):895-910.

[9] D’Souza N, Nugent K. Appendicitis. Am Fam Physician 2016;93(2):142-3.

[10] Zinner MJ, Ashely SW. Maingot's abdominal operations. Chapter-21. Appendix and appendicectomy. 11th edn. McGraw-Hill 2007:589-612.

[11] Flum DR, Koepsell T. The clinical and economic correlates of misdiagnosed appendicitis: nationwide analysis. Arch Surg 2002;137(7):799-804.

[12] Singh SK, Kunal, Chitrangda. Comparative study of diagnostic accuracy of modified Alvarado score and ultrasonography in acute appendicitis. IOSR Journal of Dental and Medical Sciences (IOSR-JDMS) 2014;13(1):36-40. 
[13] Puylaert JB. Acute appendicitis: US evaluation using graded compression. Radiology 1986;158(2):355-60.

[14] Ooms HWA, Koumans RKJ, Ho Kang YPJ, et al. Ultrasonography in the diagnosis of acute appendicitis. British Journal of surgery 1991;78(3):315-8.

[15] Franke C, Bohner H, Yang Q, et al. Ultrasonography for diagnosis of acute appendicitis: results of a prospective multicenter trial. Acute Abdominal Pain Study Group. World J Surg 1999;23(2):141-6.
[16] Douglas CD, Macpherson NE, Davidson PM, et al. Randomised controlled trial of Ultrasonography in diagnosis of acute appendicitis, incorporating the Alvarado score. Brit Med J 2000;321(7266):919.

[17] Vignault F, Filiatrault D, Brandlt ML, et al. Acute appendicitis in children: evaluation with USG. Radiology 1990;176(2):501-4. 